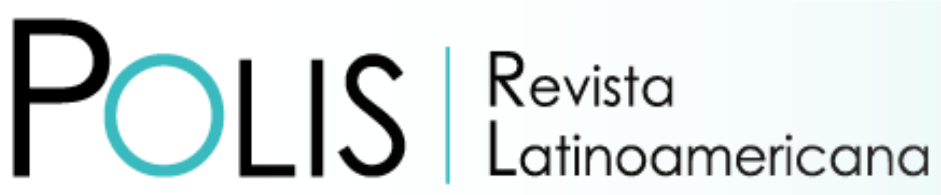

V20 | N59 | 2021

DOI: $10.32735 /$ S0718-6568/2021-N59-1591

\title{
Universidades generizadas y mercantilizadas. Implicancias para las mujeres trabajadoras en tiempo de pandemia
}

\author{
Lury Soledad Reyes Pérez \\ Universidad del Bío-Bío, Concepción, Chile \\ Email: lureyes@ubiobio.cl \\ Carmen Gloria Burdiles Cisternas \\ Universidad del Bío-Bío, Concepción, Chile \\ Email: cburdiles@ubiobio.cl \\ Jessica Carolina Jerez Yánez \\ Universidad del Bío-Bío, Concepción, Chile \\ Email: jjerez@ubiobio.cl \\ Ana Zazo Moratalla \\ Universidad del Bío-Bío, Concepción, Chile \\ Email: azazo@ubiobio.cl
}

Recibido: 20.11 .2020

Aceptado: 18.03.2021

\begin{abstract}
Resumen: Este artículo busca conocer las experiencias de las mujeres trabajadoras de una universidad pública y estatal, en un espacio laboral generizado y mercantilizado, en el contexto de pandemia. Para esto, a través de una metodología cualitativa, se desarrollaron cuatro grupos focales con participación de académicas, administrativas y trabajadoras a honorarios. El análisis realizado constata la presencia de estructuras patriarcales que reifican las relaciones tradicionales de género, un agobio y sobrecarga que afecta a todas. Sin embargo, las experiencias son disímiles, dependiendo de la calidad contractual y funciones que se realizan en su interior. Finalmente, se propone a las universidades la elaboración de políticas sensibles al género, para mitigar las consecuencias de la pandemia en las trabajadoras.
\end{abstract}

Palabras Claves: Género; covid 19; neoliberalismo; patriarcado; educación superior.

\section{Gendered and Commodified Universities. Implications for Women Working in Times of Pandemic.}

Abstract: This article seeks to know the experiences of women working in a public state university, a gendered and commercialized workplace, in the context of a pandemic. For this, through a qualitative methodology, four focus groups were developed with the participation of academic, administrative and contingent workers. The analysis carried out confirms 
the presence of patriarchal structures that reify traditional gender relations, a burden and overload that affects everyone. However, the experiences are dissimilar, depending on the contract and corresponding responsibilities. Finally, the article proposes universities develop gender-sensitive policies to mitigate the consequences of the pandemic on female workers.

Keywords: Gender; covid 19; neoliberalism; patriarchy; higher education.

\title{
Universidades generalizadas e mercantilizadas. Implicâncias para mulheres trabalhadoras em tempos de pandemia.
}

\begin{abstract}
Resumo: Este artigo busca conhecer as experiências de mulheres trabalhadoras de uma universidade pública e estadual, em um espaço laboral generalizado e mercantilizado, em contexto de pandemia. Para isso, através de uma metodologia qualitativa, foram desenvolvidos quatro grupos focais com a participação de profissionais acadêmicas, administrativas e trabalhadoras horistas. A análise realizada constata a presença de estruturas patriarcais que reificam as relações tradicionais de gênero, um fardo e uma sobrecarga que afetam a todas. No entanto, as experiências são díspares, dependendo da qualidade contratual e das funções nele desempenhadas. Finalmente, se propõe às universidades a elaboração de políticas com perspectiva de gênero, para mitigar as consequências da pandemia sob as trabalhadoras.
\end{abstract}

Palavras chave: Gênero; Covid 19; neoliberalismo; patriarcado; educação superior.

\section{Como citar este artículo:}

Reyes Pérez, L., Burdiles Cisternas, C., Jerez Yánez,J. y Zazo Moratalla,A. (2021). Universidades generizadas y mercantilizadas. Implicancias para las mujeres trabajadoras en tiempo de pandemia. Polis Revista Latinoamericana, 20 (59), 39-58. doi: http://dx.doi.org/10.32735/ S0718-6568/2021-N59-1591

\section{Introducción}

La incorporación de las mujeres a la universidad, en tanto espacio de trabajo, ha sido gradual ya que desde sus orígenes fue pensada solo para hombres (Acker, 1994), espacio que además ha experimentado profundas transformaciones tras la instalación de un modelo económico neoliberal (Sisto 2017). En este sentido, la universidad corresponde a un espacio laboral generizado y mercantilizado que, para las mujeres trabajadoras, se encuentra marcado por condiciones de desigualdad que hacen compleja su estabilidad y su desarrollo laboral y económico (Hidalgo, 2014).

En otro orden de ideas, las diversas medidas sanitarias, adoptadas por los países para hacer frente a la pandemia causa del Covid-19 develaron y profundizaron, las consecuencias de la mercantilización de lo público y el mercado como eje regulador de la vida, en especial en las mujeresenelámbito delempleoylasobrecargadeltrabajoreproductivo (CEPAL,2020). Durante la pandemia, las universidades han intentado responder a la crisis con lógicas de mercado y 
acciones neutrales al género (AUR, 2020). Sin embargo, lo neutral siempre se construye desde lo masculino invisibilizando las experiencias de las mujeres, por lo tanto, las desigualdades de género también se han profundizado en las universidades durante la pandemia.

En ese sentido, la literatura científica emergida de la pandemia da cuenta de una disminución de la productividad académica en distintos campos disciplinares como la economía (Amano-Patiño, Faraglia, Giannitsarou, y Hasna, 2020), las ciencias biológicas, las matemáticas, la física, la computación y la biología (King y Frederickson, 2020). Además, Minello (2020) concluye que, esta disminución de la productividad afectará el desarrollo académico de las mujeres si las universidades no consideran el desequilibrio existente entre hombres y mujeres en relación al cuidado familiar y al trabajo. En una línea similar, estudios como el de Malisch et al. (2020) plantean que, las universidades deben desarrollar políticas sensibles al género basadas en la equidad e indagar en las consecuencias que tendrá la pandemia en la productividad académica.

En síntesis, la mayoría de los estudios enfocan su análisis en las consecuencias de la pandemia en las académicas, desde la perspectiva de la desigual distribución del trabajo reproductivo entre hombres y mujeres. Campos (2020) es la única que aborda los impactos de la pandemia en mujeres académicas, administrativas y trabajadoras por horas (honorarios) a través de preguntas realizadas de manera "informal" (Campos, 2020, p.9). Los resultados se exponen en genérico, como mujeres trabajadoras, dando cuenta de un cansancio acumulado, percepción de jornadas laborales más extensas, con o sin la presencia de hijos en el hogar. Pero hasta ahora, no se ha realizado un trabajo sistemático que compare las percepciones entre esos grupos de mujeres trabajadoras.

Este trabajo recoge elementos de Campos (2020) y a partir de una metodología más sistematizada analiza las experiencias que el trabajo a distancia en contexto de emergencia sanitaria genera en mujeres trabajadoras en una universidad estatal. Resulta significativo observar lo que ocurre en este espacio laboral dada su centralidad en el desarrollo del pensamiento crítico respecto de la sociedad y su responsabilidad que le cabe en la construcción democrática y equitativa del país.

Situándose desdelosaportesteóricos delfeminismomaterialista queilustranlarelaciónentre trabajo productivo, doméstico y de cuidado. El recorrido metodológico será de corte cualitativo, con participación de mujeres trabajadoras que voluntariamente aportan a la investigación. Esta opción se sustenta en la necesidad de visibilizar las experiencias de sujetas históricamente subordinadas sin distingo de su relación contractual con la institución. Desde ahí, generar condiciones que posibiliten la construcción de políticas afirmativas hacia las trabajadoras.

Para dar cuenta del proceso se ha organizado este artículo en tres etapas. La primera, presenta el marco teórico y una contextualización de las disparidades de género en la universidad. En la segunda sección, se describe el método investigativo utilizado para la recopilación de información dando paso posterior al análisis. En la tercera, hacemos las discusiones y por último se presentan las principales conclusiones. 


\section{Marco teórico La división sexual del trabajo}

La división sexual del trabajo es la distribución social de actividades según se trate de hombres o mujeres. Esta distribución varía en cada sociedad y a lo largo de la historia, pero se caracteriza por estar siempre impregnada de una valoración diferenciada que jerarquiza, estructurando y organizando la vida social en todos los ámbitos, con importantes implicancias en las condiciones de vida, de hombres y mujeres. De este modo, la división sexual del trabajo, como categoría de análisis, permite por un lado comprender las formas específicas y concretas de desigualdades entre mujeres y hombres, y por otro, explicar cómo estas desigualdades operan en el nivel de las prácticas.

Analizar la presencia de las mujeres en el mercado laboral desde las dicotomías público-privado y/o productivo-reproductivo es insuficiente (Brunet Icart y Santamaría Velasco, 2016). Por ello, se recurre a una perspectiva feminista materialista que entiende que las bases estructurales que explican esta situación son la apropiación y el control de las actividades reproductoras que realizan las mujeres, trabajo del cuidado y trabajo doméstico, por parte del capitalismo (Brunet Icart y Santamaría Velasco, 2016; Delphy, 1987).

En el año 2016, la actividad económica de las mujeres tiene su mayor concentración en el sector de comercio (22,1\%), seguido de Enseñanza $(14,2 \%)$ y Salud $(9,1 \%)$. Los hombres, en tanto, se concentran principalmente en el sector comercio (17.5\%), Construcción (13,9\%), Agricultura y Pesca (12,2\%) e Industria Manufacturera (12,2\%). Lo anterior, muestra que en Chile los trabajos relacionados con la fuerza - valor asignado a lo masculino-son realizados por hombres, y aquellos relacionados con el cuidado y tareas del hogar-valores asignados a lo femenino- continúan siendo realizados por mujeres.

Por otra parte, estudios sobre uso del tiempo en Chile muestran que las mujeres realizan gran parte del trabajo doméstico y de cuidados, independientemente de su situación conyugal, laboral o de ingresos (PNUD, 2010). Las mujeres entre 25 y 59 años de edad dedican 21 horas a la semana al cuidado de personas y los hombres un 9.4 (ENUT, 2016). Finalmente, la presencia de mujeres en el plano productivo, está supeditada a las responsabilidades en el ámbito de la reproducción y las posibilidades de conciliar sus tiempos para la realización de ambas jornadas: la productiva y la reproductiva.

\section{Mujeres en la universidad como espacio de trabajo}

En el ámbito internacional, diversos estudios dan cuenta de las desiguales condiciones de trabajo entre hombres y mujeres en las universidades, y de la persistencia en las formas de dominación y desigualdad del orden patriarcal. Un estudio cuantitativo realizado en Estados Unidos muestra que, las actividades semanales que realizan las académicas tienen mayor probabilidad de estar vinculadas con la docencia, mientras que las que realizan los académicos tienen mayor probabilidad de estar relacionadas con tareas de investigación. 
Además, las mujeres reciben un 3,4\% más de solicitudes de actividades de trabajo que los hombres (O'Meara, Kuvaeva, Nyunt, Waugaman, y Jackson, 2017). Otros estudios revelan que la participación en actividades de servicio, que contribuyen al funcionamiento de las universidades, como comités, comisiones de escuela, o departamento, es mayor para las mujeres (Bird, Litt, y Wang, 2004; Guarino y Borden, 2017). Esta participación reduce el tiempo disponible para que las mujeres desarrollen actividades que gozan de mayor prestigio y beneficios como la investigación y la docencia (Bird et al., 2004).

En Chile, estudios sobre las relaciones de género al interior de las universidades, muestran una mayor presencia de mujeres entre el personal administrativos de servicio y de apoyo en relación a los hombres, y una menor presencia en el personal académico (Arce, 2018; Eytel, Rojas, Aedo, y Vivallo, 2020; Universidad Austral de Chile, 2016). Además, se constata una menor participación de académicas en las jerarquías más altas. Otro estudio analiza la participación de mujeres en la gestión de las universidades públicas chilenas durante el año 2014 y concluye que sólo el 24,3\% de los cargos directivos están ocupados por mujeres (del Pino Arriagada, Vallejos Cartes, Améstica-Rivas, y Cornejo-Saavedra, 2018). Esta participación se concentra en decanaturas o direcciones de departamento y disminuye en los niveles superiores.

En cuanto a la maternidad y las mujeres académicas, un estudio concluye que para las mujeres la maternidad es un problema para su carrera laboral, ya que las torna menos competitivas y exitosas de acuerdo a la estructura de incentivos de la universidad (Sanhueza Díaz et al., 2020). En una línea similar, otro estudio cualitativo que aborda la construcción de subjetividad en académicas, las participantes expresan dificultad para conciliar trabajo y familia (Martínez-Labrín y Bivort-Urrutia, 2014).

En síntesis, la evidencia internacional y nacional apunta a que la desigualdad de las mujeres al interior de las universidades es un problema extendido. La división sexual del trabajo estructura tanto las relaciones como la organización del trabajo de un modo que condiciona a las mujeres a trabajar desde lo reproductivo, mientras que los hombres lo hacen desde lo productivo. Además, el sistema parental y relacional de estas personas, resultó condicionante a la hora de acceder a un empleo y de ascender en la movilidad social.

\section{El capitalismo en su fase neoliberal en las universidades}

El neoliberalismo es un modelo económico que surge durante la década del 70 tras la crisis del capitalismo industrial, dando lugar a una serie de reformas para mantener el ritmo de acumulación previo a la crisis. Este modelo instala las lógicas del mercado en todos los ámbitos de sociedad, a nivel de sus estructuras productivas y normas institucionales, pero también a nivel subjetivo (Fardella, Sisto y Jiménez, 2015). De esta manera, el modelo neoliberal genera una tendencia a la flexibilidad y precarización de las condiciones laborales (Gómez \& Jódar, 2013), reduce el papel del Estado y convence a los sujetos de que son poseedores de libertad, ocultando el hecho de que en realidad forman parte 
de una cadena de producción de mercancías y de acumulación de capital (Crespo y Serrano, 2011). En Chile, este proceso de reestructuración de la economía capitalista se inicia durante la dictadura cívico-militar (1973-1990). Posteriormente, los distintos gobiernos democráticos, profundizaron dicho modelo (Leyton, 2008).

El proceso de instalación de la lógica de mercado en el ámbito de la educación inició en el año 1981. Lo anterior, dio lugar, a un tipo de financiamiento basado en criterios de demanda y endeudamiento vía crédito privados y un aumento en el número de universidades existentes, de ocho en 1981 a 57 para en 2019 (Mineduc, 2019). En este contexto, el Estado promueve la descentralización y diversificación de las fuentes de financiación universitarias debiendo competir por los recursos, mejorando su eficiencia y calidad de acuerdo al modelo (Sisto, 2017). Adicionalmente, se modifica la gestión del recurso humano, flexibilizando contractualmente a los y las trabajadores e incorporando sistemas de evaluación e indicadores de productividad, en particular para el personal académico (Sisto, 2005; Villa, 2011). En síntesis, las universidades en Chile han experimentado profundas transformaciones en base a esta nueva gestión de lo público que pretende imprimir la eficiencia y agilidad del mercado, al Estado. (Zabala-Villalón y Vidal-Molina, 2019). Sin embargo, la instalación del modelo neoliberal en la universidad, tiene una particularidad, que es que opera también como un dispositivo de reproducción y mantenimiento de dicho orden (Fardella et al., 2015; Sisto, 2017).

\section{Materiales y métodos}

Esta investigación se sitúa desde una perspectiva feminista denominada Punto de Vista Feminista (Haraway, 1995) que cuestiona el supuesto de neutralidad en la investigación y concibe la construcción de conocimiento como un proceso situado e inseparable de la posición social en la trama de relaciones sociales en las que estamos inmersas quienes investigamos, y de nuestras posiciones de dominación y subordinación, lo que nos permitirá producir conocimiento situado. Este enfoque resulta particularmente pertinente en este estudio toda vez que las autoras estamos insertas y somos parte del contexto de una universidad pública y estatal en que se lleva a cabo la investigación. En este sentido, los conocimientos situados siempre poseen una marca, un punto de vista. La decisión de indagar la situación de las mujeres trabajadoras, excluyendo a los hombres, se explica por un lado, dada la situación histórica y cultural de que en ellas recae el trabajo reproductivo, norma cultural, que también se reproduce en la sociedad chilena, se intensifica en el contexto Covid-19, tal como muestra la evidencia empírica mostrada anteriormente; y por otro, dada la decisión del equipo de investigadoras, de incidir políticamente en la generación de condiciones favorables al género.

Mediante una metodología cualitativa, de tipo descriptiva, buscamos comprender los fenómenos, analizando desde el punto de vista de sus participantes (Guerrero, 2016). Se invitó a colaborar a mujeres que realizan labores académicas y administrativas, con contrato permanente y a honorarios. Ellas, manifestaron su voluntad y consentimiento informado 
mediante formulario Google, enviado por correo electrónico. La técnica de recolección de información fue la de grupo focal, que busca comprender los sentidos que para las sujetas tiene la acción, la experiencia vivida de las sujetas (Canales, 2006).

Se llevaron a cabo dos ciclos de grupos focales. Para el primer grupo focal del ciclo inicial, consideró la diversidad en los roles institucionales y las circunstancias vitales de cotidianeidad, de acuerdo a la distribución de la Tabla 1.

Para el segundo grupo focal que convocó a las mujeres con roles directivos o de liderazgo (6), buscando representar las distintas áreas del quehacer institucional. De esta forma, la conformación del grupo quedó como sigue: representante de funcionarias/os no académicos (1), representante de académicas/os (1), directora de doctorado (1), Facultad de Ingeniería (1), Facultad de Ciencias (1), Facultad de Ciencias Empresariales (1)

Una vez avanzado el proceso de recolección y análisis, se realizó una segunda fase, donde se llevaron a cabo 2 grupos focales de profundización, en base al criterio de relación contractual. El grupo focal 1 de este ciclo, se conformó de 4 mujeres con convenio a honorarios, todas con jefatura de hogar; mientras que el grupo focal 2 fue compuesto por 7 mujeres con contrato a plazo fijo, 2 de ellas con hijos en edad pre-escolar y otras 2 como responsables principales en términos económicos.

Tabla 1:

Distribución de participantes grupo focal

\begin{tabular}{|c|c|c|}
\hline \multicolumn{2}{|l|}{ SEGÚN ROL O FUNCIÓN } & $\begin{array}{l}\text { SEGÚN CONDICIÓN DE VIDA } \\
\text { (PARA CADA GRUPO DE TRABAJADORAS }\end{array}$ \\
\hline Administrativas con contrato a plazo fijo & 3 & \multirow{4}{*}{$\begin{array}{l}\text { - Una con al menos } 1 \text { hija/o en edad preescolar. } \\
\text { - Una a cargo del cuidado de terceras personas } \\
\text { no hija/o } \\
\text { - Una con Jefatura de hogar. }\end{array}$} \\
\hline Profesionales con contrato a plazo fijo & 3 & \\
\hline Académicas con contrato a plazo fijo & 3 & \\
\hline Funcionarias con convenio a honorarios & 3 & \\
\hline
\end{tabular}

Fuente: Elaboración propia con datos de la investigación

Todos los grupos focales se realizaron a través de la plataforma Zoom y fueron registrados mediante la opción "grabar", existiendo un respaldo audiovisual del mismo. En ambas fases, el recorrido metodológico implicó: transcripción literal del grupo focal, selección y segmentación de los textos escritos, identificación de códigos manifiestos presentes en el relato de las participantes, agrupación de las categorías por tipo, y análisis de las categorías obtenidas (Navarro y Díaz, 2009). 
El primer ciclo de grupo focal se realizó cuando el trabajo a distancia llevaba tres meses de implementación en la institución y la mayor parte de las comunas de la Provincia de Concepción se encontraban con cuarentena preventiva y toque de queda nocturno. El segundo ciclo se implementó tras seis meses de trabajo a distancia y en circunstancias similares a la primera vez.

\section{Resultados}

En este apartado presentamos los resultados del análisis, para lo cual proponemos cuatro momentos relacionados con el proceso de categorización descrito en el apartado metodológico. El primero, da cuenta del contexto previo de desigualdades en que se enmarca la experiencia del trabajo a distancia en pandemia; el segundo, muestra las transformaciones experimentadas por las trabajadoras en relación a las tareas de cuidado en el contexto de trabajo a distancia; el tercero muestra la afectación que genera el trabajo remunerado producto de la modalidad a distancia en pandemia; finalmente, un cuarto momento revela las tensiones de conciliación experimentadas por las participantes en el periodo.

En cada momento exponemos diversos extractos provenientes de los grupos focales, los que han sido interpretados a partir de los objetivos de esta investigación. Estos extractos se destacan en cursiva para distinguirlos de los análisis realizados.

Las universidades desde sus inicios y hasta hace un siglo atrás eran instituciones masculinizadas, y la presencia de mujeres, como estudiantes o trabajadoras, se reprodujo bajo las mismos códigos del mundo exterior, esto es reproduciendo la división sexual del trabajo, lo que queda reflejado en la distribución de mujeres y hombres en las distintas disciplinas, lo que se conoce como segregación horizontal y en el acceso a cargos de mayor prestigio, conocido como segregación vertical (Buquet, 2013). En este sentido la Universidad del Bío-Bío no queda fuera de esa realidad, como lo expresa una de las participantes de esta investigación:

"...soy de la facultad de ingeniería, y es bastante machista, yo en mi departamento fui la primera profesora a jornada completa y hasta hace ocho años era la única con jornada completa con 15 hombres... y a nadie parecía llamarle la atención..." (Académica)

Adicionalmente y de manera transversal se reconocen prácticas machistas naturalizadas entre pares y colegas. En opinión de las participantes, estas prácticas fueron visibilizadas, a partir de las demandas de los movimientos feministas en Chile, durante mayo del 2018. Sin duda, la irrupción de los movimientos feministas, con un discurso identitario feminista, crítico contra la estructura patriarcal (Segato, 2003), se introdujo en las aulas y pasillos universitarios de Chile, logrando instalar la desigualdad de género como un asunto de carácter político institucional en las universidades: 
"...nos hemos ido dando cuenta de que había cosas que estaban normalizadas en nuestro trato diario (...), nosotras teníamos que asumir que era un mundo de hombres y que los tratos eran así ..." (Académica)

Un aspecto central del proceso de neoliberalización de las universidades, es la transformación del trabajo académico a niveles que se correspondan con el management. Desarrollando procesos de evaluación del desempeño según ciertos estándares, incentivos por resultados, entre otros (Fardella et al., 2015) que invisibilizan las inequidades de base.

"En el espacio universitario no solamente se trabaja para formar personas, sino que también uno tiene que producir muchas cosas. Y así es como nos miden, y acá está muy instalado, como dijeron las colegas, el tema de los artículos..., es difícil (...) cumplir con todos los indicadores con que te miden" (Académica)

Menor presencia, una situación de exclusión caracterizada por la segregación disciplinaria y prácticas cotidianas de machismo y una nueva universidad con una racionalidad neoliberal, en palabras de las participantes, dan cuenta de una institución organizada de acuerdo a la lógica de género y del mercado. Así nos encontró la pandemia y es en este contexto que las trabajadoras de la Universidad deben llevar adelante los desafíos del trabajo a distancia en el contexto de crisis sanitaria.

\section{Trabajo de cuidados}

Sin lugar a dudas, el cuidado, entendido este como todo aquello que se hace para mantener y reparar el mantenimiento cotidiano del entorno, la vida, el bienestar físico y emocional y todo lo necesario para el sostenimiento de la vida (Arriagada, 2010a) adquirió una visibilidad y centralidad sin precedentes desde el inicio de las medidas de confinamiento (CEPAL, 2020). En este sentido, la pandemia dejó en exposición la crisis de los cuidados, particularmente en aquellos países cuya resolución está principalmente en privados, como es el caso chileno (Arriagada, 2010b).

Para las mujeres trabajadoras, lo que antes presentaba la posibilidad de ser distribuido entre varias instituciones y/o personas (escuelas, empleadas de casa particular), en el contexto de pandemia ha recaído principalmente en ellas. Las mujeres realizan varios trabajos remunerados y no remunerados en un mismo espacio y tiempo difusos, lo que ha tenido efectos tanto en la extensión de la jornada laboral como en la percepción de mayor ansiedad y estrés, al igual que lo encontrado por Campos (2020). Sin embargo, el mandato cultural de género, que exige que estas tareas sean realizadas por las mujeres, está presente en los discursos de las entrevistadas:

"Trabajo desde las 12 del día hasta las 3 de la mañana, ya sea haciendo mis labores formales de la universidad, como labores el hogar, porque como mujer nos toca dirigir eso de la casa" (Académica) 
Situación especial es el caso de las mujeres que han debido asumir el ejercicio del trabajo remunerado y crianza de hijos/as, como únicas cuidadoras, lo que sin duda incrementa la demanda de las labores de cuidado. Lo anterior, en algunos casos llevó al desarrollo de estrategias para disminuir la sobrecarga, que no siempre resultaron exitosas.

Otro de los aspectos que componen las tareas de cuidado son de tipo afectivo y emocional (Arriagada, 2010a), en este sentido, existe preocupación por cómo la situación actual afecta a sus hijos/as, sintiéndose responsables de contenerlos emocionalmente. Sentimientos que se extienden hacia sus parejas y progenitores. Estos últimos, al ser parte de la población de riesgo, constituyen una preocupación importante para las mujeres, pero también una oportunidad en cuanto a la profundización de los vínculos familiares:

"ha sido el periodo en que más he hablado con mi papá, algo muy raro (...) una emoción nueva que se forma en mi vida y una tensión, siento un poco una responsabilidad por apoyarlo" (Administrativa)

Así también, manifiestan haber estrechado vínculos de colaboración y ayuda con las personas de su entorno más inmediato, es decir vecinos y vecinas. Así como la canalización de ayudas y cooperaciones monetarias para apoyar a quienes están más afectados por la crisis.

"Cuando tengo que ir al supermercado, yo le hago las comprar a mi vecina porque su esposo está postrado entonces yo me comunico con ella y le llevó" (Honorarios).

Las personas que habitan solas también han requerido de rutinas, pues muchas veces asumen labores de cuidado con terceros, principalmente padres/madres, abuelas/abuelos, además de sobrellevar el confinamiento con una mayor "distancia social" y una red de apoyo más reducida.

\section{Trabajo productivo}

Con relación al trabajo productivo, si bien existen aspectos comunes, es posible encontrar algunas diferencias en la experiencia del trabajo a distancia en tiempos de pandemia, dependiendo de la relación contractual y funciones que se realicen en la Universidad. Lo común dice relación con que la situación de confinamiento produce sensación de ambivalencia en las participantes: por una parte, un malestar por la obligada complementariedad de roles productivo y reproductivo, y por otra, la satisfacción de poder estar en familia y en su hogar, protegidas de los riesgos de la pandemia:

"Si a mí me preguntan si yo prefiero estar en la Universidad trabajando o prefiero estar en mi casa en tiempo de pandemia, prefiero estar trabajando en la casa trabajando extra y aguantando el estrés." (Administrativa) 
Otro aspecto compartido por la totalidad de las participantes es que existe una percepción de aumento de carga laboral respecto del trabajo desarrollado con anterioridad a la crisis sanitaria. Transversalmente predomina la idea de que la jornada laboral se alarga en el intento diario de compatibilizar trabajo remunerado con el de cuidados:

"El primer mes yo nunca me acosté antes de las tres de la mañana (...) y a las nueve de la mañana estaba en reunión(...) hubo un momento súper importante que me puse a llorar y exploté (...) ni siquiera un día había podido hacerles almuerzo a mis hijos" (Académica)

En el caso de trabajadoras administrativas, ya sea de contrata o a honorarios, los límites de la jornada laboral se tornan aún más difusos; las funcionarias transitaron de una jornada marcada por una hora de inicio y término, con un estricto control de asistencia, a una jornada que parece no tener inicio ni término, donde el control se ejerce mediante una excesiva demanda de reportes diarios por parte de jefaturas. De esta manera, el modelo de gestión y control de los recursos que requiere el modelo neoliberal para evidenciar eficiencia, demanda un excesivo flujo de reportes, que se traduce en agotamiento y malestar en las mujeres.

En el contexto de la pandemia, las labores de apoyo a estudiantes y docentes adquieren una mayor notoriedad, las universidades despliegan estrategias para contener a estudiantes y evitar deserción, un criterio de eficiencia en el actual modelo universitario. Esto produce una sensación de injusticia o inequidad por parte del sistema y su estructura, acentuada por una sobre exigencia personal por cumplir y "ser responsables" con las y los estudiantes y el trabajo.

En este sentido, Giannini (2020) evidencia la particularidad de las funciones del personal no docente, pues no asoman como la actividad principal de la Universidad, pero al ser de soporte, resultan vitales para el funcionamiento de esta. Hay una sensación de deshumanización del trabajo remunerado, lo que ha generado desgaste sobre todo a nivel de salud mental, haciéndose un llamado a que la institución ponga atención respecto a la presión en que se encuentra trabajando el plantel profesional y de administración, en este contexto.

"entiendo la necesidad de sostener esto, pero también estamos cansados y así, (...) la tónica que está llevando este proceso es súper intenso, súper profundo y está siendo súper poco humano" (Honorarios)

En lo que dice relación con las mujeres académicas, surge un llamado a la universidad a adoptar medidas de conciliación de las responsabilidades laborales y la flexibilización de los indicadores de rendimiento laboral en el contexto de la actual crisis sanitaria. Se observa un cuerpo de mujer, tensionado por dos sistemas de dominación, el neoliberal y el patriarcado: el primero presionando por productividad y el segundo respondiendo al mandato de género: 
"Yo no estoy pidiendo una consideración especial por ser mujer, estoy pidiendo igualdad de condición. Porque siento que mi tiempo, mis ocho horas, yo no puedo producir lo mismo que un hombre en ocho horas, porque yo estoy en ese tiempo preocupándome de mi hijo, cosa que el hombre no lo hace" (académica)

En cuanto a las condiciones materiales del trabajo a distancia, encontramos diferencias según el tipo de contrato. Académicas y administrativas han podido trasladar equipos y mobiliarios a sus domicilios para realizar sus actividades. Sin embargo, en el caso de las trabajadoras a honorarios, algunas no contaban con un computador para realizar sus actividades laborales, ni antes ni durante la pandemia. En algunos casos el acceso dependía de la voluntad de la jefatura:

“...nunca tuve un computador en la universidad, yo llevaba mi notebook al trabajo (...) ahora, por la situación de la pandemia se hizo más urgente poder tener un computador y me prestaron uno, por una gestión que realizó el coordinador de mi programa..." (Honorarios)

La precariedad laboral dada por la ausencia de un contrato, da cuenta de las consecuencias del proceso de neoliberalización de las universidades (Communes, 2017) y que se agudiza durante la pandemia. Otra consecuencia de esta precariedad laboral, es una mayor preocupación por la continuidad laboral, debido al riesgo que la pandemia ha implicado para la estabilidad económica de las universidades, dada su actual estructura de financiamiento.

Una práctica habitual en la docencia universitaria es la circulación entre universidades, realidad que aparece con la mercantilización de las universidades, que recurre a la subcontratación de servicios, como forma de ocultar la precarización (Zabala-Villalón y Vidal-Molina, 2019). Sin embargo, las trabajadoras a honorarios que realizan labores de docencia, resaltan la oportunidad de disponer de mayor tiempo para trabajar o cuidar de la familia, debido a la ausencia de desplazamientos entre el hogar y universidad. De esta manera, la pandemia ha reducido los tiempos de desplazamiento entre universidades a tan solo dos "click", uno de salida y otro de entrada, amplificando la productividad de los cuerpos que dependen de sí mismos para mejorar su productividad.

\section{Conciliación}

De acuerdo con Haar, Russo, Suñe y Ollier-Malaterre (2014) la conciliación trabajo-familia, se basa en la percepción que cada sujeto o sujeta tiene en relación al equilibrio de su vida laboral y otros roles que desempeña en su vida, lo que dependerá de sus propias circunstancias y necesidades.

En este sentido, lo más complejo del trabajo a distancia, ha sido encontrar un equilibrio en la compatibilización de las labores de trabajo remunerado y responsabilidad de cuidado de hijos/as. La sensación de no encontrar suficientes alternativas para lograr un equilibrio saludable, resulta ser un sentimiento transversal para las mujeres entrevistadas, sobre todo en 
el caso de aquellas que cumplen roles de cuidado a niñas, niños y adolescentes. La emergencia sanitaria ha implicado el despliegue de roles en forma simultánea, de este modo las mujeres se sienten tensionadas por las demandas del ámbito público y del ámbito privado, ya que todo ocurre en el mismo tiempo y espacio.

"Yo he estado en reuniones de dos, tres horas y parece que no saben que una tiene que hacer otras cosas, claro como son puros hombres." (Académica)

Las mujeres han procurado acomodar sus rutinas diarias en pro del trabajo. Relatan haber modificado sus dinámicas cotidianas, partiendo por la distribución del tiempo y horarios, hasta llegar a la alteración de espacios físicos. No todas disponen de espacio físico para habilitar oficina, lo cual implicó utilizar comedores, dormitorios, etc. para dichos fines. El trabajo a distancia a causa del confinamiento, instaló lo público y lo privado en los hogares de las mujeres, sin considerar las condiciones de habitabilidad ni equipamiento que estas disponían y menos aún las estructuras y dinámicas familiares existentes, solo llegó y se instaló.

"...mi casa ya no es el espacio donde yo iba a relajarme, llegaba al final del día y hacía otras cosas que no tenían que ver con el trabajo, ahora tengo que sacar la mesa, todo, meterlo dentro de un cajón, para sentir que no estoy en mi trabajo". (Honorarios)

En los casos donde hay hijos/as, el escenario es más complejo y la complementariedad de los roles paternos es más urgente. En las participantes se evidencia apoyo y la presencia de compañeros medianamente "colaboradores". Toda vez que en chile sigue siendo una actividad que recae principalmente en las mujeres (ENUT, 2016):

"tenía un horario en la oficina (...), trabajaba, me iba a cierto horario y traté de aplicarlo acá en mi casa y al principio me costó un montón porque entre el rol de mamá, de dueña de casa, de profesora que soy de los niños, todo, no me daba, no tenía tiempo de hacerme cargo de todos los roles..." (Administrativa)

La mujer se predispone a las labores múltiples ejecutadas en paralelo. En cambio, el hombre sigue asumiendo como su principal desafío el cumplir con el rol proveedor y "colaborar" en la medida de lo posible con lo doméstico. Se evidencia en esto la fuerte influencia del modelo imperante y la mirada patriarcal en torno a las tareas domésticas, lo que no se cuestiona mayormente desde las participantes.

Adicionalmente, las nuevas formas que adquiere el trabajo a distancia en tiempos de pandemia, se llevan a cabo, bajo el supuesto de que quienes participan en ellas responden a un sujeto hombre que tiene la domesticidad resuelta. En este sentido, pareciera ser que, para algunos, estar en la casa debido al confinamiento, implicaría disponer de más tiempo, situación que, en el caso de las mujeres, no es así. Esto genera una tensión y cansancio adicional en las mujeres trabajadoras: 
"Yo he estado en reuniones de dos, tres horas y parece que no saben que una tiene que hacer otras cosas, porque en la universidad jamás tuvimos una reunión de tres horas, jamás. Las reuniones son de una hora. Ahora es como que una tuviera todo el tiempo del mundo para la reunión y no es así" (Académica)

Por otra parte, para varias entrevistadas, especialmente aquellas con hijos pequeños, describen una demanda desde el sistema escolar, que asumen acríticamente, que tensiona aún más a las mujeres trabajadoras y que en ocasiones de crisis frente al fracaso escolar interpela al otro, al que está en la periferia de los cuidados:

\footnotetext{
"Ilenan la plataforma de tareas y tengo que hacerla yo con él, y, ¿̇en qué minuto? (...) tengo yo que sentarme, a ver el video, para entender cómo puedo ayudarlo, y aparte de eso tengo que preparar el material didáctico, o sea, entonces, no. No me da. Entonces otro día le dije a mi esposo que, por mí, si repite, repite. Y mi esposo: "¿Cómo va repetir?" y yo le dije "¿'Tú has hecho algo para que no repita?". (académica)
}

El trabajo doméstico y de cuidados que se realiza en el hogar, asignado tradicionalmente a las mujeres, impacta negativamente en el ámbito laboral remunerado, ya que requiere de tiempo y energías, que se le resta al desarrollo de actividades de trabajo remunerado. Acker y Dillabough (2007) encontraron que las académicas madres experimentaron importantes grados de ansiedad, cansancio y falta de sueño como problemas asociados a la combinación del trabajo académico con el doméstico y el de crianza. Sin embargo, la necesidad de responder a los estándares de productividad laboral, especialmente entre las académicas, las lleva a desplegar estrategias como trabajar por más tiempo o en momentos que antes destinaban al descanso. Esto en respuesta a las altas expectativas de productividad, especialmente en lo que refiere al ámbito de la investigación y publicaciones, que han orientado el quehacer de la universidad en los tiempos que hoy corren.

En todas las participantes, asoman emociones duales, por cuanto se valora el contar con la oportunidad y tiempo de asumir la labor de cuidado, pero a su vez asumen esta como desgastante. Este hallazgo confirma la afirmación de (Lagarde, 2016) en cuanto a que las mujeres trabajadoras viven en un estado contradictorio opresivo-liberador en el cual el conflicto es explicado y vivido tanto con angustia como con incapacidad, disgusto o culpa.

\section{Discusiones y conclusiones}

La pandemia encontró una universidad orientada en las lógicas de la eficiencia y de mercado, situación exacerbada, tras los procesos de reestructuración, en particular aquellos relativos a la racionalización productiva implementados en Chile a partir de la década de los 80. Dentro de esta estructura y desde sus orígenes, el trabajador concebido en este espacio, es el de un cuerpo abstracto y masculino, concepción que a través de una lógica de la división sexual del trabajo opera como criterio de organización del espacio laboral. 
Lo descrito en el párrafo anterior es percibido por las mujeres participantes del estudio. Es algo que logran identificar y que les genera malestar, pero al mismo tiempo, se aprecia un poderoso arraigo cultural que se les presenta como un mandato difícil de subvertir. Esto es más evidente en el grupo de mujeres académicas, quienes se ven sometidas a los criterios de productividad que recaen sobre la academia.

En relación al grupo de trabajadoras con contratos parciales o por horas que participaron del estudio, se observa una mayor precariedad en las condiciones materiales básicas para el desarrollo del trabajo a distancia, así como también una mayor sensación de incertidumbre frente a su continuidad laboral, dada la condición de precariedad. Esta sensación, pese a ser experimentada por ellas en circunstancias de no pandemia, en el transcurso de la misma se incrementa.

En cuanto al trabajo doméstico y de cuidados, de acuerdo a lo relatado por las participantes, ha aumentado su visibilidad en términos del tiempo e intensidad que demandan, lo que antes presentaba la posibilidad de ser distribuido entre varias instituciones y personas (escuelas, empleadas de casa particular, redes de apoyo de cuidados), en el contexto de pandemia ha recaído principalmente en las mujeres. Esto es aún más complejo en el grupo de mujeres que tiene hijos pequeños o en edad escolar donde no se cuenta con la presencia del padre o pareja, o estando no asume un rol activo en estas funciones.

Entendemos que las universidades no podrán hacerse cargo de las condicionantes estructurales que recaen sobre el cuerpo de las mujeres. Sin embargo, creemos es imperativo que estas hagan todos los esfuerzos por conocer y analizar los efectos que esta crisis tiene para el desempeño laboral de las mujeres trabajadoras y disminuir las consecuencias negativas que la pandemia está teniendo para las mujeres que realizan trabajo a distancia.

Por otra parte, las desigualdades no son inevitables, sino que son producto de formas particulares de relaciones que lo organizan (Rubin, 1986). En este sentido, las universidades tienen una mayor responsabilidad, frente a la desigualdad de género, toda vez que se trata de un espacio que cultiva en su interior el pensamiento crítico respecto de la sociedad y son las llamadas a realizar propuestas en cuanto a una construcción democrática y equitativa del país. Es por ello que la crisis pandémica, puede abrir espacios de oportunidad para reflexionar sobre las relaciones sociales en su interior y realizar transformaciones, que permitan el desarrollo y reconocimiento de todos quienes aportan a los propósitos institucionales. Rompiendo con la reproducción de la desigualdad al resto de la sociedad.

En procesos investigativos posteriores, sería interesante revisar los efectos que esta crisis sanitaria y sistema de trabajo a distancia, pudiera generar en hombres trabajadores, en el marco de estudio de nuevas masculinidades. 


\section{Agradecimientos}

Este artículo es parte del proyecto de investigación I+D 20-49 "Mujeres y trabajo a distancia en tiempos de COVID-19: análisis y monitoreo en Universidad del Bío-Bío, sede Concepción", financiado por la Universidad del Bío-Bío.

\section{Bibliografía}

Acker, S. (1994). Género y Educación, Reflexiones sociológicas sobre mujeres, enseñanza y feminismo. Madrid: Ediciones Narcea.

Acker, S., y Dillabough, J.-A. (2007). Women 'learning to labour'in the 'male emporium': Exploring gendered work in teacher education. Gender and Education, 19(3), 297-316. doi: https://doi.org/10.1080/09540250701295460

AUR. (2020). UNIVERSIDADES REGIONALES: Aportes y Experiencias ante la Situación Generada por la Pandemia de Coronavirus (COVID-19).

Amano-Patiño, N., Faraglia, E., Giannitsarou, C., y Hasna, Z. (2020). Who is doing new research in the time of COVID-19? Not the female economists. En S. Galiani \& U. Panizza (Eds.), Publishing and Measuring Success in Economics. London: Centre for Economic Policy Research. Recuperado de: https://voxeu. org/article/who-doing-new-research-time-covid-19-not-female-economists

Arce, J. (2018). Análisis sobre las relaciones de género en la Universidad de Valparaíso. Valparaiso, Chile: Universidad de Valparaiso, Unidad de Igualdad y Diversidad. Recuperado de: https://igualdadydiversidad.uv.cl/attachments/ article/56/An\%C3\%A 1 lisis\%20sobre\%20las\%20relaciones\%20de\%20genero\%20 UV.pdf

Arriagada, I. (2010a). La crisis de cuidado en Chile. Revista de Ciencias Sociales, (27), 58-67. Recuperado de: https://www.redalyc.org/pdf/4536/4536461 14006.pdf

Arriagada, l. (2010b). La organización social de los cuidados y vulneración de derechos en Chile. Santiago: ONU Mujeres-CEM. Recuperado de http://www. cem. cl/pdf/cuidadoschile. pdf.

Batthyány, K., y Sánchez, A. S. (2020). Profundización de las brechas de desigualdad por razones de género: El impacto de la pandemia en los cuidados, el mercado de trabajo y la violencia en América Latina y el Caribe. Astrolabio, (25), 1-21. Recuperado de: https://revistas.unc.edu.ar/index.php/astrolabio/ article/view/29284

Bird, S., Litt, J., y Wang, Y. (2004). Creating status of women reports: Institutional housekeeping as" Women's Work". NWSA Journal, 194-206. doi: 10.1353/ nwsa.2004.0027

Botello, N. A. (2013). Perspectivas teóricas de la violencia: Modos epistémicos. Fermentum. Revista Venezolana de Sociología y Antropología, 23(66), 33-56. Recuperado de: https://www.redalyc.org/pdf/705/70538668003.pdf

Brunet Icart, I., y Santamaría Velasco, C. A. (2016). La economía feminista y la división sexual del trabajo. Culturales, 4(1), 61-86. Recuperado de: http://www. scielo.org.mx/scielo.php?pid=\$1870-11912016000100061 \&script=sci_arttext

Brunner, J. J. (1999). La educación superior frente a los desafíos del futuro. Discurso de Inauguración del Año Académico (Valparaíso: Universidad de Valparaíso).

Buquet, A. (2013). Sesgos de Género en las Trayectorias Académicas Universitarias: Orden cultural y estructura social en la división sexual del trabajo (Tesis Doctoral). Universidad Nacional Autónoma de México, México DF, México. Recuperado de: https://www.ses.unam.mx/integrantes/uploadfile/rrodriguez/Buquet2013_Tesis.pdf

Buquet, A. (2014). El peso de las identidades de género en la reproducción de las desigualdades en instituciones de educación superior. En M. Rifà Valls, L. Duarte Campderrós, y M. Ponferrada Arteaga (Eds.), Nuevos Desafíos para la Inclusión Social y la Equidad en la Educación Superior Actas del III Congreso Internacional MISEAL, noviembre, 2014. (p. 165). 
Campos, C. P. (2020). De académicas, pandemia, encierro y bitácoras: Experiencias de algunas universitarias en el contexto del COVID-19. Revista Reflexiones, 99(2). doi: https://doi.org/10.15517/rr.v99i2.42271

Canales, M. (Ed.). (2006). Metodologías de la investigación social (Primera). Santiago de Chile: LOM ediciones.

Cárdenas, A., Correa, N., y Prado, X. (2014). Segregación laboral y género: Tendencias y desafíos relativos al mercado laboral de la salud y la educación en Chile. Polis. Revista Latinoamericana, (38). Recuperado de: https://journals. openedition.org/polis/10182

CEPAL, N. (2020). La pandemia del COVID-19 profundiza la crisis de los cuidados en América Latina y el Caribe. CEPAL. Recuperado de: https://repositorio. cepal.org/handle/11362/45335

Communes, C. (2017). El gobierno Neoliberal de la Universidad en Chile. Colectivo Communes. De Prácticas y Discursos, 6(8), 89-104. doi: DOl: http://dx.doi. org/10.30972/dpd.682372

Cooper, J. (2014). ¿Por qué la meritocracia traiciona a las mujeres académicas en las instituciones de educación superior? Análisis y propuestas. Calidad de la educación superior y género en América Latina, 131. Recuperado de: https://www.researchgate.net/profile/Ana-Buquet-Corleto-2/ publication/286450069_Genero_y_educacion_superior_una_mirada_desde_ America_Latina/links/5669f1 d708äe62b05f0275d0/Genero-y-educacion-superior-una-mirada-desde-America-Latina.pdf\#page $=133$

Crespo, E., y Serrano, A. (201 1). Regulación del trabajo y el gobierno de la subjetividad: La psicologización política del trabajo. Perspectivas recientes y críticas en la Psicología Social actual, Madrid: Biblioteca Nueva. Recuperado de: http://webs.ucm.es/centros/cont/descargas/documento25372.pdf

del Pino, S., Cartes, R., Rivas, L., y Saavedra, E. (2018). Presencia de las mujeres en la alta gestión universitaria. Las universidades públicas en Chile. Páginas de educación, 11 (2), 176-198. Recuperado de: https://dialnet.unirioja.es/servlet/ articulo? codigo $=6761520$

Delphy, C. (1987). Modo de producción doméstico y feminismo materialista. Mujeres, ciencia y práctica política, Madrid: Debate.

Díaz Arias, D., y Viales Hurtado, R. (2020). Centroamérica: Neoliberalismo y COVID-19. Central América: Neoliberalism and COVID-19., 11, 53-59. doi: https:// doi.org/10.5209/geop.69017

ENUT. (2016). Encuesta Nacional Sobre Uso del Tiempo. Documento de Principales Resultados ENUT 2015 (p. 69). Santiago de Chile: Instituto Nacional de Estadísticas. Recuperado de: https://historico-amu.ine.cl/enut/files/principales_resultados/documento_resultados_ENUT.pdf

Eytel, B., Rojas, C., Aedo, C., y Vivallo, O. (2020). Diagnóstico de situación y relaciones de género en la Universidad de La Frontera. (p. 243). Temuco, Chile: Universidad de La Frontera. Recuperado de: https://www.ufro.cl/ images/UFRO_AL_DIA/2020/diciembre/24/Diagnostico-Situacion-y-Relaciones-de-Genero-en-UFRO.pdf

Fardella, C., Sisto, V., y Jiménez, F. (2015). Nosotros los académicos. Narrativas identitarias y autodefinición en la universidad actual. Universitas Psychologica, 14 (SPE5), 1625-1636. doi: https://doi.org/10.11144/Javeriana.upsy14-5. nani

Fazackerley, A. (2020). Women's research plummets over lockdown-but articles from men increase. The Guardian May. Recuperado de: https://www. theguardian.com/education/2020/may/12/womens-research-plummetsduring-lockdown-but-articles-from-men-increase

Federici, S. (2013). Revolución en punto cero: Trabajo doméstico, reproducción y luchas feministas. Madrid: Traficantes de sueños.

Fernández, A. R. (2020). El tiempo de las mujeres: Trabajo y malestar femenino en tiempos de pandemia. Revista Reflexiones, 99(2). doi: 10.15517/rr.v99i2.42150

Fisher, B., y Tronto, J. (1990). Toward a feminist theory of caring. Circles of care: Work and identity in women's lives, 35-62.

Flaherty, C. (2020). No room of one's own: Early journal submission data suggest COVID-19 is tanking women's research productivity. Inside Higher Ed, 21. Recuperado de: https://www.insidehighered.com/news/2020/04/21/early-journal-submission-data-suggest-covid-19-tanking-womens-research-productivity 
King, M. M., y Frederickson, M. (2020). The Pandemic Penalty: The gendered effects of COVID-19 on scientific productivity. Recuperado de: https://www. uni-konstanz.de/typo3temp/secure_downloads/109827/0/315ee3b807d83fblad1977f86e86345f49361605/King_Frederickson_2020_Pandemic_Penalty. pdf

Giannini, S. (2020). COVID-19 y educación superior: De los efectos inmediatos al día después. Revista Latinoamericana de Educación Comparada, 11 (17), 1-57. Recuperado de: https://dialnet.unirioja.es/descarga/articulo/7502929. pdf

Gill, R. (2009). Breaking the silence: The hidden injuries of neo-liberal academia. Secrecy and silence in the research process: Feminist reflections, $21,21$.

Gómez Fuentealba, P. S. (2017). Género y educación profesional universitaria: Transformaciones y continuidades.

Gómez, L., y Jódar, F. (2013). Ética y política en la universidad española: La evaluación de la investigación como tecnología de la subjetividad. Athenea Digital. Revista de pensamiento e investigación social, 13(1), 81-98. Recuperado de: https://www.redalyc.org/pdf/537/53725662007.pdf

Guarino, C. M., y Borden, V. M. (2017). Faculty service loads and gender: Are women taking care of the academic family? Research in higher education, 58(6), 672-694. Recuperado de: https://link.springer.com/article/10.1007/s11162$017-9454-2$

Guerrero, M. A. (2016). La investigación cualitativa. INNOVA Research Journal, 1(2), 1-9. doi: https://doi.org/10.33890/innova.v1.n2.2016.7

Haar, J. M., Russo, M., Suñe, A., y Ollier-Malaterre, A. (2014). Outcomes of work-life balance on job satisfaction, life satisfaction and mental health: A study across seven cultures. Journal of Vocational Behavior, 85(3), 361-373. doi: https://doi. org/10.1016/j.jvb.2014.08.010

Haraway, D. J. (1995). Ciencia, cyborgs y mujeres: La reinvención de la naturaleza (Vol. 28). Universitat de València.

Hart, J. L., y Cress, C. M. (2008). Are women faculty just "worrywarts?" Accounting for gender differences in self-reported stress. Journal of Human Behavior in the Social Environment, 17(1-2), 175-193. doi: https://doi. org/10.1080/10911350802171120

Hidalgo, C. (2014). Análisis de remuneraciones y composición por género en la Universidad de Chile. Santiago. Recuperado de: http://www.uchile.cl/documentos/cev-remuneraciones-y-composicion-de-genero_113390_39_2208.pdf

INE. (2015). Mujeres en Chile y mercado del trabajo: Participación laboral femenina y brechas salariales (p. 138). Santiago, Chile: Instituto Nacional de Estadísticas. Recuperado de: https://www.ine.cl/docs/default-source/ocupacion-y-desocupacion/publicaciones-y-anuarios/publicaciones/mujeres-en-chile-y-mercado-del-trabajo---participaci\%C3\%B3n-laboral-femenina-y-brechas-salarialesa.pdf? sfvrsn=ade344d 43

Instituto Nacional de Estadística. (2017). Enfoque Estadístico de Género y Empleo (p. 15). Santiago, Chile. Recuperado de: https://historico-amu.ine.cl/genero/ files/estadisticas/pdf/documentos/enfoque-estadistico-genero-y-empleo. pdf

Lagarde, M. (2016). Los cautiverios de las mujeres: Madresposas, monjas, putas, presas y locas. Siglo XXI Editores México.

Leache, P. A., y Sordoni, L. M. (2013). Gubernamentalidad neoliberal, subjetividad y transformación de la universidad. La evaluación del profesorado como técnica de normalización. Athenea Digital. Revista de pensamiento e investigación social, 13(1), 99-120. Recuperado de: https://atheneadigital.net/article/ view/1046

Leyton, J. C. G. (2008). Política y ciudadanía en una sociedad neoliberal avanzada, Chile 1990-2007. Cuadernos del CENDES, 25 (67), 59-83. Recuperado de: http://ve.scielo.org/scielo.php? pid=S101225082008000100004\&script=sci_arttext\&tlng=pt

Malisch, J. L., Harris, B. N., Sherrer, S. M., Lewis, K. A., Shepherd, S. L., McCarthy, P. C., ... y Deitloff, J. (2020). In the wake of COVID-19, academia needs new solutions to ensure gender equity. Proceedings of the National Academy of Sciences of the United States of America, $117(27), 15378-15381$. doi: https:// doi.org/10.1073/pnas.2010636117 
Martínez-Labrín, S., y Bivort-Urrutia, B. (2014). Procesos de producción de subjetividad de género en el trabajo académico: Tiempos y espacios desde cuerpos femeninos. Psicoperspectivas, 13(1), 15-22. doi: http://dx.doi.org/10.5027/psicoperspectivas-Voll3-Issue l-fulltext-334

Mead, M. (1963). Sex and temperament in three primitive societies (Vol. 370). Morrow New York. Recuperado de: https://personalwebs.coloradocollege. edu/ mduncombe/WS\%201 10/Mead,\%20Sex\%20and\%20Temperament.pdf

Mineduc. (2019). Estadísticas de la Educación 2018. Santiago, Chile.

Minello, A. (2020). The pandemic and the female academic. Nature, 17. Recuperado de: http://covidacademics.co.za/Uploads/docs/The-pandemic-and-the-female-academic.pdf

Ministerio de Educación, Centro de Estudios. (2019). Estadísticas de la Educación 2018. Santiago, Chile. Recuperado de: https://centroestudios.mineduc.cl/ wp-content/uploads/sites/100/2019/11/ANUARIO-2018-PDF-WEB-FINALr.pdf

Mitchell, S. M., y Hesli, V. L. (2013). Women don't ask? Women don't say no? Bargaining and service in the political science profession. PS: Political Science \& Politics, 46(2), 355-369. doi: https://doi.org/10.1017/S1049096513000073

Nash, M., y Churchill, B. (2020). Caring during COVID-19: A gendered analysis of Australian university responses to managing remote working and caring responsibilities. Gender, Work \& Organization, 27(5), 833-846. doi: https://doi. org/10.1111/gwao.12484

Navarro, P., y Díaz, C. (2009). Análisis de contenido. En J. Delgado \& J. Gutiérrez (Eds.), Métodos y técnicas cualitativas de investigación en ciencias sociales. (pp. 177-224). España: Editorial Síntesis.

Oficina de Igualdad de Oportunidades de Género U. de Chile Universidad de Chile. (2013). Del biombo a la cátedra. Igualdad de oportunidades de género en la Universidad de Chile. (p. 211). Santiago: Comisión de Igualdad de Género de la Universidad de Chile. ISBN/ISSN 978-956-324-278-2 Recuperado de: http://web.uchile.cl/archivos/VEX/BiomboCatedra/files/assets/basic-ht$\mathrm{ml} /$ index.html\# 1

O'Meara, K., Kuvaeva, A., Nyunt, G., Waugaman, C., y Jackson, R. (2017). Asked more often: Gender differences in faculty workload in research universities and the work interactions that shape them. American Educational Research Journal, 54(6), 1154-1 186. doi: https://doi.org/10.3102/0002831217716767

PNUD. (2010). Informe de Desarrollo Humano en Chile. Género: Los desafíos de la igualdad (p. 363). Santiago de Chile: PNUD. Recuperado de: https://www. ocac.cl/wp-content/uploads/2015/01/ONU-MUJERES-2010-G\%C3\%A9nero-los-desaf\%C3\%ADos-de-la-igualdad.pdf

Poblete, R. (2011). Género y Educación: Trayectorias de vida para ellos y ellas Gender and Education: trajectories of life for them and they. Revista Latinoamericana de Educación Inclusiva, 63. Recuperado de: http://www.rinace. net/Rlei/numeros/vol5-numl/art4_htm.html

Reverter Bañón, S. (2020). ¿Está reventando el capitalismo neoliberal la liberación de las mujeres? Is Neoliberal Capitalism Bursting the Liberation of Women? 9(17), 193-213. Recuperado de: http://www.lastorresdelucca.org/index.php/ ojs/article/view/399/345

Ríos González, N., Mandiola Cotroneo, M., y Varas Alvarado, A. (2017). Haciendo género, haciendo academia: Un análisis feminista de la organización del trabajo académico en Chile. Psicoperspectivas, 16(2), 114-124. doi: http:// dx.doi.org/10.5027/psicoperspectivas-vol16-issue2-fulltext-1041

Rivera, M. D., y Cerda, J. I. P. (2017). Análisis del subcontrato en las universidades chilenas, especialmente en el caso de las universidades estatales, y las vías jurídicas para su término. Revista Chilena de Derecho del Trabajo y de la Seguridad Social, 8(16), 81-100. doi: 10.5354/0719-7551.2017.48032

Rubin, G. (1986). El tráfico de mujeres: Notas sobre la" economía política" del sexo. Nueva antropología, 8(30), 95-145. Recuperado de: https://www.redalyc.org/pdf/159/15903007.pdf

Sanhueza Díaz, L., Fernández Darraz, C., y Montero Vargas, L. (2020). Segregación de género: Narrativas de mujeres desde la academia. Polis. Revista Latinoamericana, (55). doi: https://doi.org/10.32735/S0718-6568/2020-N55-1453

Segato, R. (2003). Las estructuras elementales de la violencia: Ensayos sobre género entre la antropología, el psicoanálisis y los derechos humanos ( la. ed.). 
Buenos Aires: Universidad Nacional de Quilmes: Prometeo 3010. Recuperado de: https://ninosyninas.defensoria.gov.co/assets/img/mentoring/06_psicosocial/04_psicosocial.pdf

Segato, R. (2018). La guerra contra las mujeres (Segunda). Buenos Aires, Argentina: Prometeo Libros.

Sisto, V. (2005). Flexibilización laboral de la docencia universitaria y la gest (ac) ión de la Universidad sin órganos. Un análisis desde la Subjetividad Laboral del Docente en Condiciones de Precariedad. Biblioteca/sisto_subjedocl_clacso. pdf. Recuperado de http://www.psiucv.cl/wpcontent/uploads/2012/11/ Flexibiizacion Laboral_Docencia.pdf

(2017). Gobernados por números: El financiamiento como forma de gobierno de la universidad en Chile. Psicoperspectivas, 16(3), 64-75. doi: http:// dx.doi.org/10.5027/psicoperspectivas-vol16-issue3-fulltext-1086

Universidad Austral de Chile. (2016). Diagnóstico de Género y Diversidad. Etapa 1 (p. 91). Valdivia, Chile: Comite Paritario de Género y Diversidad, Universidad Austral de Chile. Recuperado de: https://www.uach.cl/uach/_file/diagnostico-gye-informe-1-v2-587d348a516a2.pdf

Villa, M. D. (2011). Los discursos sobre la flexibilidad y las competencias en la educación superior. Pedagogía y saberes, (35), 9-24. doi: https://doi.or$\mathrm{g} / 10.17227 / 01212494.35$ pys9.24

Zabala-Villalón, G., y Vidal-Molina, P. (2019). El Trabajo bajo el neoliberalismo: Subcontratación en una universidad estatal chilena. Polis (Santiago), 18(54), 268-293. https://doi.org/10.32735/S0718-6568/2019-N54-1409

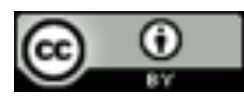

Este es un artículo de acceso abierto bajo licencia Creative Commons Reconocimiento 4.0 Internacional 